Thin-film stretchable electronics technology based on meandering interconnections: fabrication and mechanical performance

This article has been downloaded from IOPscience. Please scroll down to see the full text article.

2012 J. Micromech. Microeng. 22015002

(http://iopscience.iop.org/0960-1317/22/1/015002)

View the table of contents for this issue, or go to the journal homepage for more

Download details:

IP Address: 157.193.127.142

The article was downloaded on 09/12/2011 at 08:14

Please note that terms and conditions apply. 


\title{
Thin-film stretchable electronics technology based on meandering interconnections: fabrication and mechanical performance
}

\author{
R Verplancke, F Bossuyt, D Cuypers and J Vanfleteren \\ Centre for Microsystems Technology (CMST), ELIS Department, Ghent University-IMEC, \\ Technologiepark 914a, 9052 Gent, Belgium \\ E-mail: Rik.Verplancke@UGent.be
}

Received 15 July 2011, in final form 11 October 2011

Published 8 December 2011

Online at stacks.iop.org/JMM/22/015002

\begin{abstract}
A new fabrication technology for stretchable electrical interconnections is presented. This technology can be used to connect various non-stretchable polyimide islands hosting conventional electronic components. The interconnections are realized by patterning a $200 \mathrm{~nm}$ thick sputter-deposited gold film into meandering horseshoe shapes, functioning as 'two-dimensional springs' when embedded in a silicone elastomer. Polyimide support is introduced around the meandering conductors as a means to improve the mechanical performance. Processing is done on a temporary carrier; the islands and interconnections are embedded in polydimethylsiloxane in a final stage. To this end, a release technique compatible with high temperatures up to $350{ }^{\circ} \mathrm{C}$ based on the evaporation of a $400 \mathrm{~nm}$ thick layer of potassium chloride is developed. Test structures consisting of stretchable interconnections with a varying polyimide support width were fabricated. These were strained up to twice their original length without compromising their functionality. Also cyclic mechanical loading at various strains was performed, indicating the influence of the polyimide support width on the lifetime. At strains of $10 \%$, a minimum lifetime of 500000 cycles is demonstrated. The presented technology thus provides a promising route towards the fabrication of stretchable electronic circuits with enhanced reliability.
\end{abstract}

(Some figures in this article are in colour only in the electronic version)

\section{Introduction}

Stretchable electronics is a relatively new concept representing electronic systems that are able to cope with relatively large mechanical strains without compromising their functionality. In recent years, this field has gained widespread interest as stretchable circuits enable conformability of electronic systems to more complex 3D shapes in comparison with conventional flexible systems. They allow for improved user comfort and reliability by enhanced dynamical shaping and matched mechanical properties of the electronic system to its environment. Applications clearly benefiting from these improvements include biomedical devices such as wearable and implantable health monitors or biosensors which need to interface with soft living tissue [1]. The ability to reversibly strain electronics allows for simultaneous electrical and mechanical actuation and monitoring, enabling new opportunities in diverse multidisciplinary research areas such as stretchable microelectrode arrays [2,3].

To date, a variety of strategies towards the realization of stretchable electronic systems have been reported. Focus is put on the development of stretchable electrical interconnections as it is a key aspect for these systems (see section 2). One strategy consists in the development of conductive materials that are intrinsically stretchable $[4,5]$. Other strategies devise ingenious structural designs of conventional high-performance 


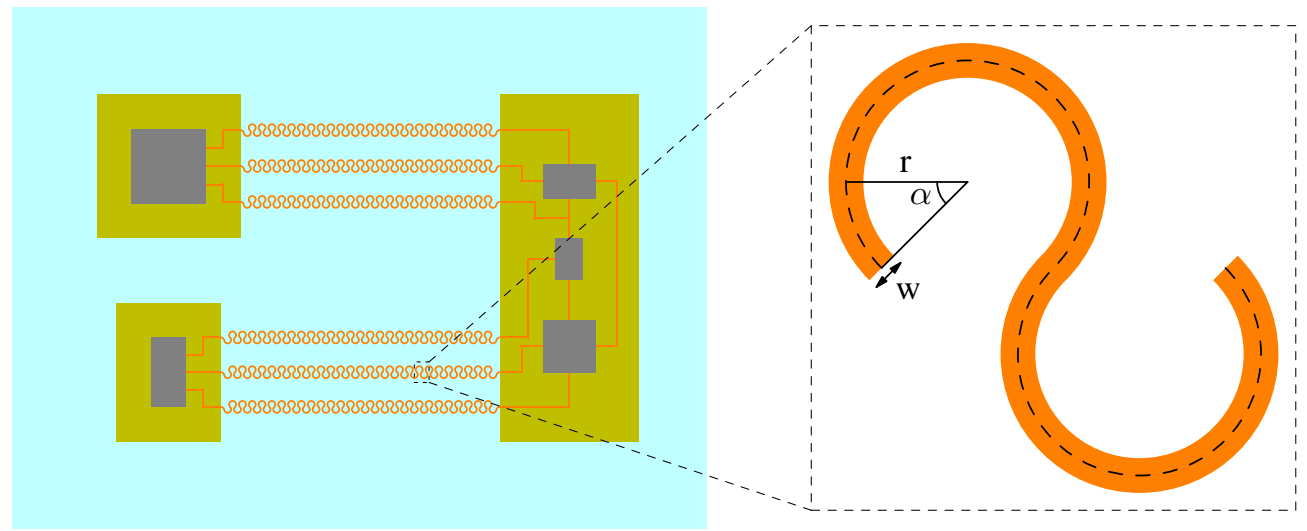

Figure 1. Stretchable circuit composed of stiff islands hosting conventional electronic components, interconnected by meandering horseshoe-shaped conductors.

electronic materials: both out-of-plane $[6,7,9]$ and in-plane [10-14] layouts of these materials bonded to or embedded in an elastomer have been reported to accommodate strains applied in the plane.

One approach uses inorganic materials that are uniformly or selectively bonded to pre-stretched polydimethylsiloxane (PDMS) membranes, resulting respectively in wavy or buckled hybrid systems upon mechanical relaxation [6, 7]. Mesh layouts with devices located at planar nodes, interconnected by non-coplanar serpentine triple-layer stacks of polymer and metal, have been used to realize complex stretchable circuits [7, 8]. A comparable approach involves evaporation of thin metal films on a PDMS membrane, resulting either in a continuous but wavy [9] or a flat but microcracked film $[10,11]$. Alternative approaches are based on the patterning of metal films into shapes that can be reversibly strained when combined with an elastomer such as PDMS [12-14].

The technology presented in this paper is based on the last approach: stretchable electrical interconnections are realized by embedding meandering conductors in PDMS. First, the concept of the presented technology is elucidated by discussing an architecture based on the meandering interconnections. Then, the fabrication process is described in detail in the experimental section; two important process steps are highlighted. Finally, the mechanical performance of fabricated test structures is discussed.

\section{Stretchable electronics concept}

As electronic components are typically rigid or in the best case flexible [15], stretchable circuits can be envisioned by connecting various stiff, non-stretchable islands through stretchable electrical wiring. The islands are composed of regions of a structural polymer with limited degree of flexibility, hosting one or more electronic components which are interconnected in a conventional way. This architecture is illustrated in figure 1.

Stretchable electrical interconnections hence form a key challenge to enable stretchable circuits. These are realized by meandering conductors that are able to function as 'twodimensional springs' when embedded in a silicone elastomer.
Based on the results of finite element analysis described in detail elsewhere [16], a horseshoe-shaped design for the conductor is proposed. The basic repetitive unit and its mechanical design parameters (angle $\alpha$, radius $r$ and width $w$ ) are schematically represented in figure 1 . Proper design ensures that the local strain magnitudes in the conductor stay below the point of rupture. This design depends on different factors such as applied deformation, track resistance and interconnection pitch. However, this will not be the topic of this paper.

The failure mechanisms of horseshoe-shaped conductors have been experimentally verified to be crack formation and crack propagation, similar to fatigue fracturing [17]. The occurrence of these phenomena can be postponed by a number of measures. Finite element analysis illustrated that decreasing the track width can tremendously decrease the magnitude of the stress levels in the conductor [16], postponing the formation of cracks. The crack propagation can be delayed by introducing a polymer material with a higher Young's modulus than PDMS, as a support for the horseshoe-shaped conductors. Furthermore, increasing the width of the support can effectively reduce the plastic strain in the metal conductor [18], again postponing the formation of cracks.

The proposed technology relies on the fabrication of reliable stretchable wiring by a proper support for the meandering conductors. It features thin-film metallization to enable a fine interconnection pitch and track width by restricting underetch.

\section{Experimental}

In what follows, a fabrication process is described which allows the simultaneous realization of stiff component islands and meandering conductor support. Polyimide PI-2611 (HD MicroSystems) is chosen as the structural polymer material, with a Young's modulus of $8.5 \mathrm{GPa}$ [19]. Furthermore, PI-2611 films exhibit other desirable properties such as relatively low moisture uptake $(0.5 \%)$, low stress and low CTE ( $3 \mathrm{ppm}{ }^{\circ} \mathrm{C}^{-1}$ ), making them ideally suited for thin-film metal deposition. Gold is chosen as the conductor material for improved biocompatibility. 


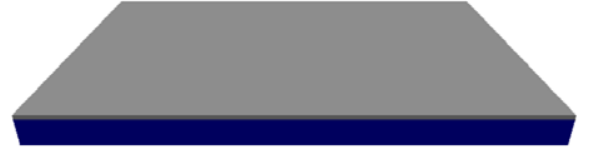

(a) Glass substrate with release stack

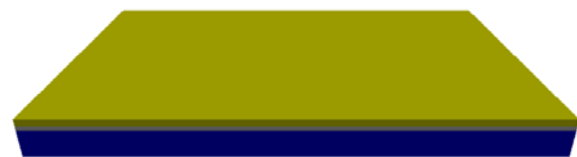

(b) Spin coat polyimide

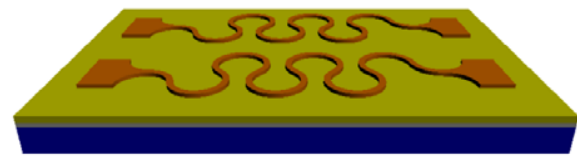

(c) Sputter deposition + lithography of

TiW/Au

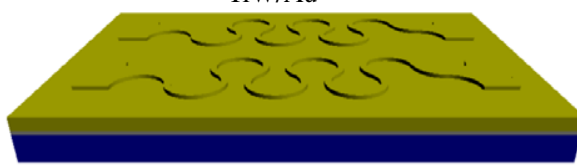

(d) Spin coat polyimide

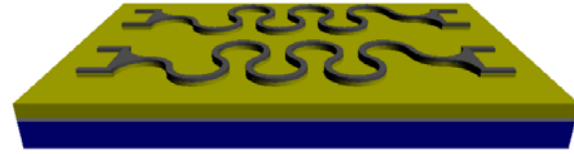

(e) Sputter deposition + lithography of TiW/Al

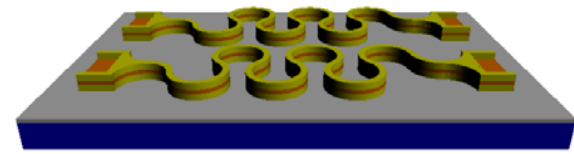

( $f$ ) Dry etch polyimide

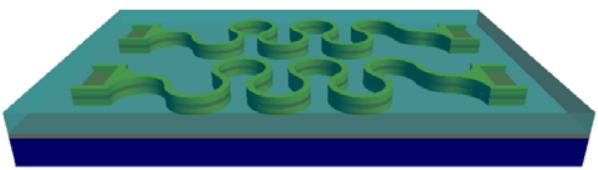

( $g$ ) Embed top side into PDMS

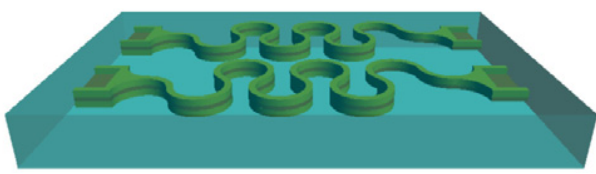

(h) Embed bottom side into PDMS

Figure 2. Schematic representation of the fabrication process.

\subsection{Fabrication process}

A schematic representation of the fabrication process is shown in figure 2. Fabrication of the stretchable circuit is done on a rigid carrier (float glass, Corning 1737F) before being embedded in PDMS. Although this facilitates different process steps and enhances alignment during photolithography, it requires the development of a customized release technology. This has been proved quite challenging and is discussed in detail in section 3.3.

After deposition of the release stack (figure 2(a)), a $5.5 \mu \mathrm{m}$ thick layer of PI-2611 is spin coated and cured at $350{ }^{\circ} \mathrm{C}$ (figure $2(b)$ ). Next, a $50 \mathrm{~nm}$ thick titanium tungsten adhesion layer and a $200 \mathrm{~nm}$ thick gold film are sputter deposited within one vacuum cycle and patterned using photolithography and wet etching (figure $2(c)$ ). After the photoresist strip in acetone, another $5.5 \mu \mathrm{m}$ thick PI-2611 film is spin coated and cured (figure $2(d)$ ).

Both supporting bottom and top polyimide films are subsequently patterned by dry etching. The top film is masked by a $200 \mathrm{~nm}$ thick aluminium film, preceded by a $50 \mathrm{~nm}$ thick titanium tungsten adhesion layer, which are sputter deposited and patterned using photolithography and wet etching (figure 2(e)). It was verified that the gold film, like the aluminium film, effectively serves as a masking layer for the dry etching, making it possible to provide contact pads to the gold conductor (as illustrated in figure $2(f)$ ) by etching the top polyimide film. Dry etching conditions depend on the etch tool and film thickness and will be discussed in more detail in section 3.4. The aluminium hard mask is finally stripped by wet etching, leaving polyimide-covered interconnections together with polyimide islands laying on top of the release stack (figure $2(f)$ ).
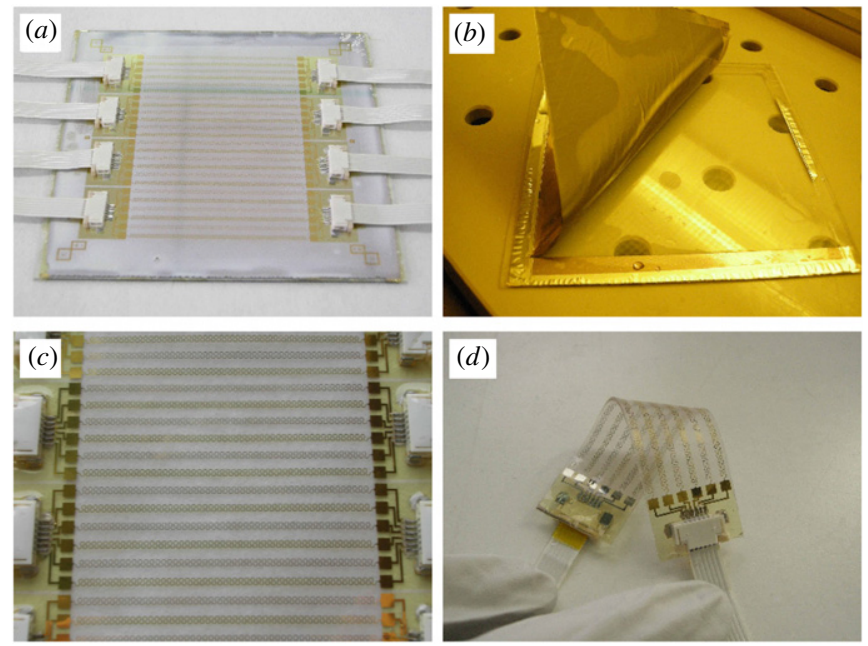

Figure 3. (a) Molex ZIF sockets are mounted on PI-2611 islands; (b) the partially embedded stretchable circuit is released from the rigid carrier; $(c)$ the sacrificial layers are etched; $(d)$ the stretchable circuit is fully embedded in Silastic ${ }^{\circledR}$ MDX4-4210 and highly conformable.

At this point, conventional Molex ZIF sockets are mounted by means of an isotropically conductive adhesive CE 3103 (Emerson \& Cuming) (figure 3(a)). This adhesive is chosen as it is found impossible to perform regular soldering without damaging the gold film because it dissolves too rapidly into the solder. Next, the stretchable circuit is embedded in PDMS (section 3.2).

\subsection{Embedding in PDMS}

Embedding in PDMS is done either by spin coating or liquid injection moulding, both in two phases to provide full 


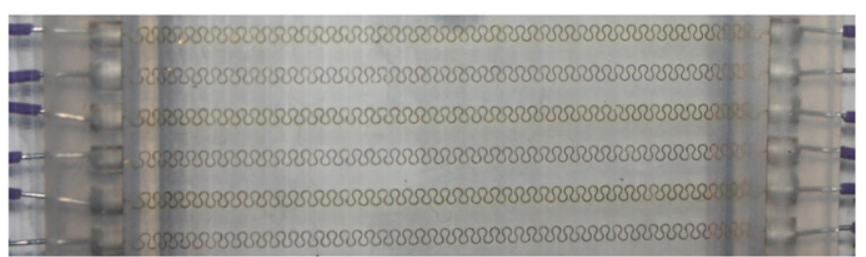

Figure 4. Stretchable interconnections embedded in Sylgard ${ }^{\circledR} 186$ by liquid injection moulding.

embedding. The former method is chosen when thin, uniform films of PDMS are desired, while for the latter method the substrate is clamped in between 2 PMMA moulds, offering the possibility of performing non-uniform 3D embedding. Both methods have been used to embed the test structures discussed in section 4, illustrating the generic character of the technology with respect to the embedding procedure.

Two types of PDMS are used, Silastic ${ }^{\circledR}$ MDX4-4210 (biomedical grade elastomer, Dow Corning) for spin coating and Sylgard ${ }^{\circledR} 186$ (Dow Corning) for liquid injection moulding. Prior to application of the PDMS, 1200 OS Primer (Dow Corning) is applied to the substrate for improved adhesion of the PDMS to the polyimide structures.

After applying the first layer of PDMS, it is cured for $4 \mathrm{~h}$ in ambient atmosphere in a convection oven at $50^{\circ} \mathrm{C}$ (figure $2(\mathrm{~g})$ ). The stack is then released from the rigid carrier (figure $3(b)$ ) and the sacrificial layers are removed (figure 3(c)) according to the procedure described in section 3.3. Subsequently, the embedding procedure is repeated at the bottom side of the released membrane (figure $2(h)$ ). The embedded test structures are shown in figure $3(d)$ and figure 4; the PDMS thickness is 1.2 and $2.5 \mathrm{~mm}$, respectively.

\subsection{Release technique}

As noted in the previous section, an appropriate release technique is required to transfer the stretchable circuit from its rigid carrier to PDMS. Release procedures typically involve the deposition of sacrificial materials which are removed at a later stage. Since the sacrificial material is present during the complete fabrication process (as illustrated in figure 2), it should not be damaged during the subsequent process steps, nor should its presence compromise the quality of the process.

Diverse sacrificial materials have already been demonstrated in the literature ranging from materials removed by wet etching, dry etching or dissolution in an appropriate solvent to thermally released temporary adhesives. Release procedures involving the former three are typically diffusion limited and thus time consuming. Anodic metal dissolution [20] has been reported to be significantly faster than its diffusion-limited chemical etching variant, but release is still quite time consuming and highly dependent on stack buildup properties such as thickness and mechanical properties. Release procedures involving thermally released temporary adhesives are typically area independent and hence preferred in this context, though none was found to be compatible with the high-temperature curing profile of PI-2611. In addition to compatibility with the high temperatures, the sacrificial materials have to be compatible with the solvent carrier n-methyl-2-pyrrolidone (NMP) used in PI-2611 and should not cause premature release during the process.

Despite the variety of release procedures, none was found fully complying with the above-mentioned fabrication process. Therefore, a customized release procedure is developed. Potassium chloride $(\mathrm{KCl})$ is chosen as a base material since it is chemically inert, has a melting point of $772{ }^{\circ} \mathrm{C}$ and can easily be released at a later stage by dissolution in deionized water. A uniform film is deposited by thermal evaporation using a Leybold Heraeus Univex 450 deposition system. To prevent premature stack release during the wet processing steps described in section 3.1, a shadow mask is used to prevent potassium chloride from being deposited on the edges of the carrier. Next, a $5.5 \mu \mathrm{m}$ thick film of PI-2611 is spin coated on top of this layer and ensures effective shielding against moisture penetration after curing.

To determine the right thickness, different thicknesses of deposited potassium chloride ranging from 50 to $400 \mathrm{~nm}$ were investigated by means of scanning electron microscopy (SEM). Depending on the deposited thickness, a different surface topography of the $\mathrm{KCl}$ layer is obtained. The SEM image of a $100 \mathrm{~nm}$ thick layer is shown in figure 5(a), clearly indicating the presence of pinholes. These pinholes result in local adhesion of the subsequently deposited PI-2611 film to the rigid carrier and will later on hamper a successful release. Figure $5(b)$ shows a $400 \mathrm{~nm}$ thick layer where no pinholes are detected, which will eventually result in a successful release.

As the sacrificial polyimide layer should not be attacked during the structuring of the polyimide layers supporting the stretchable interconnections (figure $2(f)$ ), an additional $50 \mathrm{~nm}$ titanium tungsten adhesion layer and $1 \mu \mathrm{m}$ aluminium film are sputter deposited to serve as an etch stop between the structural and sacrificial polyimide films. Sputter deposition of these metal films directly on top of the potassium chloride was found to be ineffective as the shielding layer during the wet processing steps.

Since the release stack thus consists of three layers, release and stripping are performed in several phases. First, an incision is made according to the desired outline for the stretchable circuit through the complete release stack. Next, the substrate is placed in a beaker with deionized water to dissolve the $\mathrm{KCl}$. The stack is then easily separated from its rigid carrier (figure $3(b)$ ), followed by backside etching of the shielding polyimide and aluminium films respectively by dry and wet etching according to the procedures described in section 3.1.

In figure 6, test patterns are shown which are transferred to Silastic ${ }^{\circledR}$ MDX4-4210 using the presented release procedure. Meandering interconnections with a track width down to $20 \mu \mathrm{m}$ were successfully transferred.

\subsection{Dry etching of PI-2611 to form the meandering conductor support}

Dry etching conditions of polyimide typically depend on the etch tool and also on processing details such as deposited 

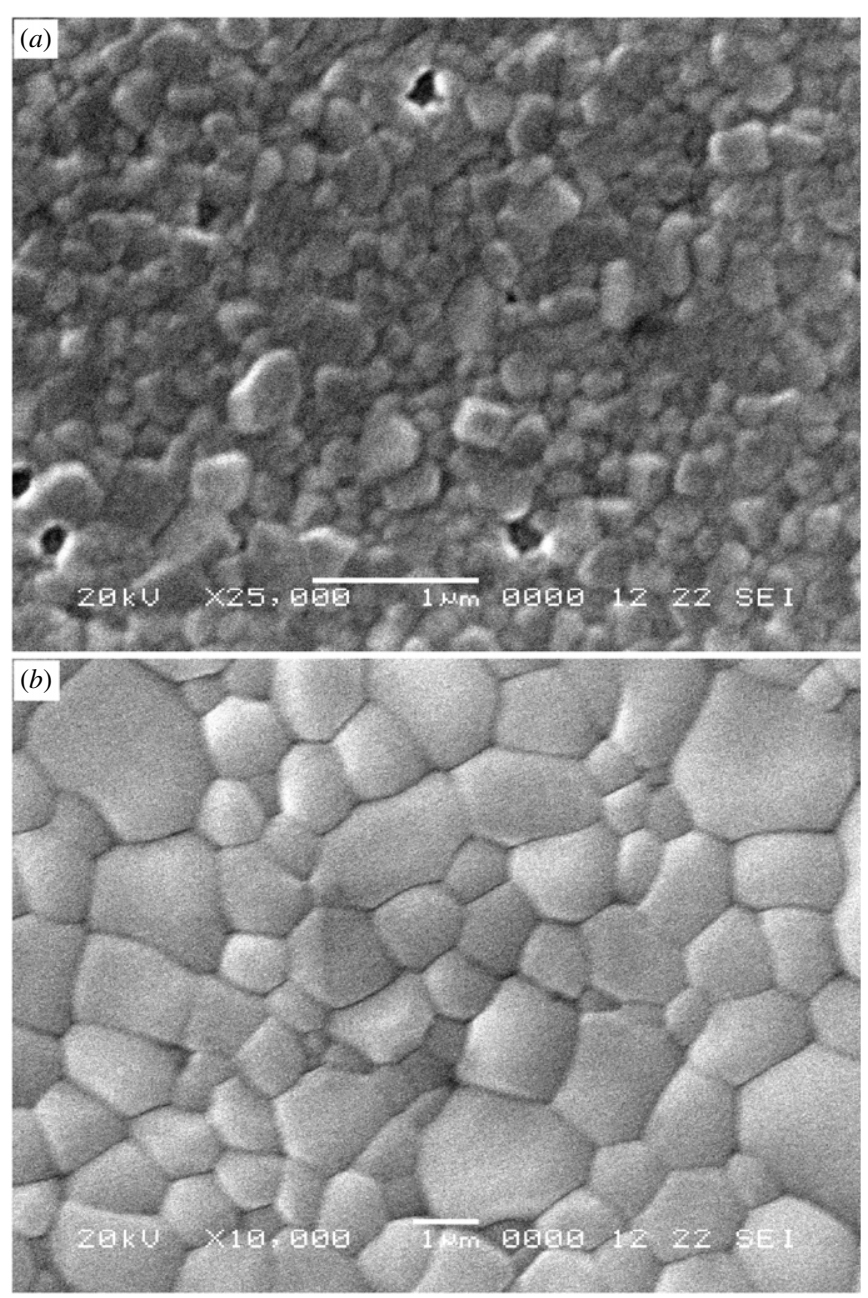

Figure 5. Surface topography of evaporated potassium chloride with different thicknesses: $100 \mathrm{~nm}(a)$ and $400 \mathrm{~nm}(b)$.

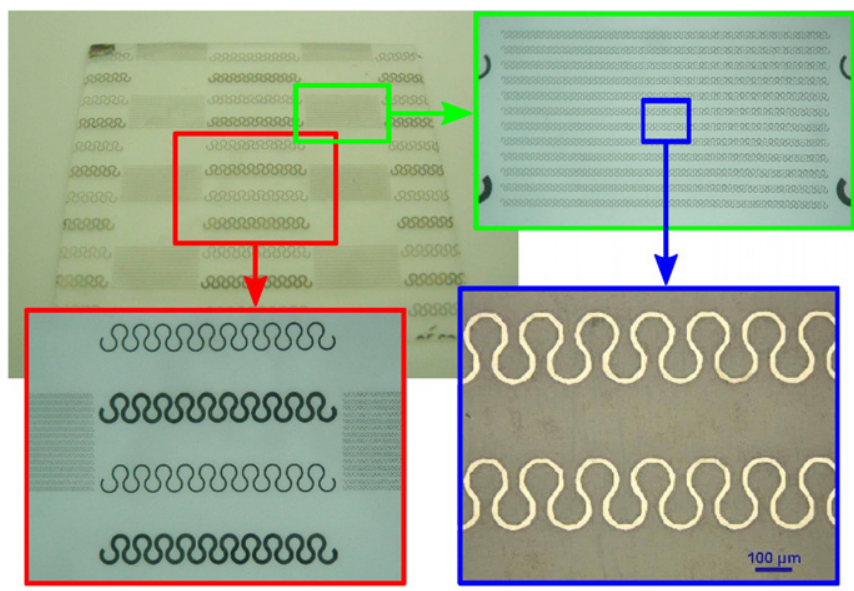

Figure 6. Meandering interconnections in Silastic ${ }^{\circledR}$ MDX4-4210; the track width in the pictures on the right is $20 \mu \mathrm{m}$ (scale bar: $100 \mu \mathrm{m})$.

film thickness and curing profile. Therefore, an optimization of the different dry etching parameters is performed based on a design of experiments (DOE). Running through all of the possible etch processes, as is the case with a full factorial
Table 1. The two levels and corresponding values of the dry etching parameters.

\begin{tabular}{llll}
\hline Parameter & Symbol & Level 1 & Level 2 \\
\hline $\mathrm{O}_{2}: \mathrm{CHF}_{3}$ flow rates (sccm) & $\mathrm{A}$ & $15: 5$ & $25: 5$ \\
$\mathrm{RF}$ power (W) & $\mathrm{B}$ & 100 & 200 \\
Chamber pressure (mTorr) & $\mathrm{C}$ & 75 & 150 \\
\hline
\end{tabular}

Table 2. Experiments performed based on an $\mathrm{L}_{4}\left(2^{3}\right)$ orthogonal array; dry etching of the PI-2611 films was performed for 5 min.

\begin{tabular}{|c|c|c|c|c|c|}
\hline \multirow[b]{3}{*}{ Experiment no } & \multirow{2}{*}{\multicolumn{3}{|c|}{$\begin{array}{c}\text { Parameter } \\
\text { level }\end{array}$}} & \multicolumn{2}{|c|}{ Response } \\
\hline & & & & \multirow{2}{*}{$\begin{array}{l}\text { Etch depth } \\
(\mu \mathrm{m})\end{array}$} & \multirow{2}{*}{$\begin{array}{l}\text { Etch uniformity } \\
(\mathrm{nm})\end{array}$} \\
\hline & A & B & $\mathrm{C}$ & & \\
\hline 1 & 1 & 1 & 1 & 0.87 & 4.90 \\
\hline 2 & 1 & 2 & 2 & 1.92 & 12.2 \\
\hline 3 & 2 & 2 & 1 & 1.57 & 13.4 \\
\hline 4 & 2 & 1 & 2 & 1.29 & 8.90 \\
\hline
\end{tabular}

experiment, requires a quantity of experiments which increases exponentially with the number of process parameters. Such a basic DOE is generally both time consuming and expensive to implement.

The Taguchi method [21] designs the experiments based on orthogonal arrays and allows for the study of a large parameter space with a limited number of experiments. It is used to predict the optimal combination of process parameters. Finally, a confirmation experiment is conducted to verify this prediction.

Three parameters are selected for optimization: RF power, chamber pressure and $\mathrm{O}_{2}: \mathrm{CHF}_{3}$ flow rates, with the constraint that the $\mathrm{CHF}_{3}$ flow rate is kept constant at $5 \mathrm{sccm}$. A two-level orthogonal array is constructed to discover trends in the dry etching. The parameter values are based on values and ranges mentioned in the PI-2611 product data sheet [19]; these are listed in table 1. Evaluation is done with respect to the etch rate and etch uniformity over a surface of approximately $5 \mathrm{~cm}$ by $5 \mathrm{~cm}$.

PI-2611 films with a thickness of $5.5 \mu \mathrm{m}$ were prepared by spin coating on glass substrates. A $200 \mathrm{~nm}$ thick aluminium film was sputter deposited and lithographically patterned. After the photoresist strip, dry etching of the PI-2611 films during 5 min was conducted using a System VII Batchtop RIE (Plasma-Therm). Finally, the aluminium hard mask was wet etched. Each process step was performed in accordance with section 3.1.

The etch depth was measured using an optical profiler WYKO NT3300 (Veeco). This was done at five welldistributed spots along the outline of a cross, one spot at the centre of the substrate and four spots at approximately $5 \mathrm{~mm}$ from each of the substrate edges, and an average is calculated. The etch uniformity is quantified by calculating the RMS value related to these etch depths; a smaller RMS value implies a better etch uniformity. Both values are mentioned in table 2 for the performed experiments. Figure 7 shows the trend lines of each parameter with respect to the etch depth and the etch uniformity, i.e. the evolution of the evaluated quantity when 


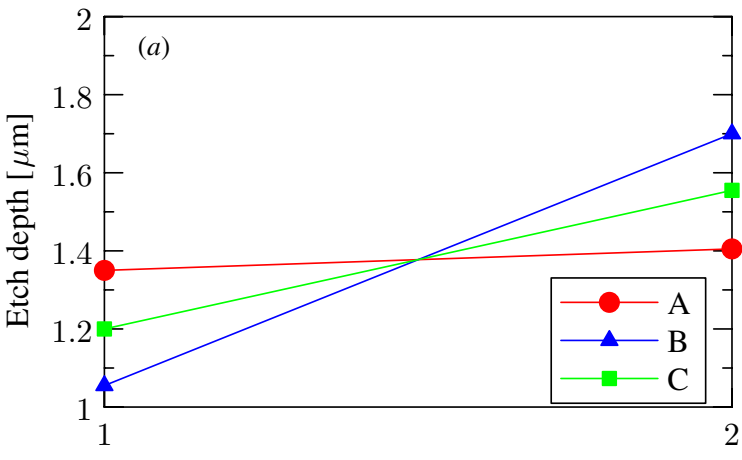

Parameter level

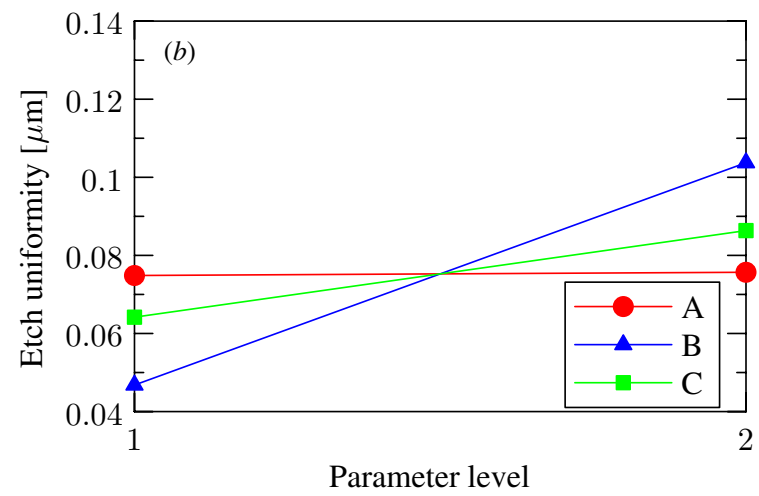

Figure 7. Trend lines for the dry etching parameters with respect to $(a)$ the etch depth and $(b)$ the etch uniformity.

the respective parameters are altered. It is noticed that the RF power is the most important parameter (largest slope) for both the etch depth and the etch uniformity, followed by the chamber pressure and the $\mathrm{O}_{2}: \mathrm{CHF}_{3}$ flow rate ratio (smallest slope). Increasing any of the parameters results not only in an increased etch rate, but also in a decreased etch uniformity. Hence, a trade-off analysis was made keeping in mind the significance of the different parameters. Levels 1.5, 2 and 1 are selected, corresponding respectively to an RF power of $150 \mathrm{~W}$, a chamber pressure of $150 \mathrm{mTorr}$ and $\mathrm{O}_{2}: \mathrm{CHF}_{3}$ flow rates of $15: 5 \mathrm{sccm}$. The analysis was also used to predict an average etch depth of $1.53 \mu \mathrm{m}$ and etch uniformity of $8.59 \mathrm{~nm}$. Experimentally, these were determined respectively as $1.65 \mu \mathrm{m}$ and $6.57 \mathrm{~nm}$, closely corresponding to the predicted values.

\section{Mechanical performance}

To illustrate the capabilities of the presented technology, test structures composed of horseshoe-shaped interconnections designed along straight parallel lines were fabricated. These were then used as a means to evaluate the mechanical performance of the stretchable interconnections. Onetime uniaxial stretching (section 4.2) is performed on test structures embedded by spin coating (figure $3(d)$ ), while cyclic mechanical loading (section 4.3) is performed on test structures embedded by liquid injection moulding (figure 4).

\subsection{Design of the test structures}

The design of the test structures is illustrated in figures 3 and 4. The horseshoe-shaped interconnections have a point-to-point length of approximately $45 \mathrm{~mm}$, and the design parameters indicated in figure 1 were fixed at $\alpha=45^{\circ}, r=350 \mu \mathrm{m}$ and $w=100 \mu \mathrm{m}$. These parameters were not optimized in terms of mechanical performance, but are well suited as a general indication. Furthermore, the line-to-line pitch, defined as the distance between corresponding points on adjacent tracks, was designed to be $3.15 \mathrm{~mm}$ in order to minimize the effect of the interconnection pitch on the mechanical behaviour of the stretchable interconnections [22].

As the integration of polyimide support for the meandering conductors is a key feature of the presented technology, two different widths of the polyimide support were included in the design to illustrate its effect on the mechanical performance. Polyimide support widths of $100 \mu \mathrm{m}$ (same width as the conductor) and $200 \mu \mathrm{m}$ (100 $\mu \mathrm{m}$ wider than the conductor) were alternately included in the design. A zoomed view of the fabricated interconnections can be seen in figure 8.

\subsection{Electrical resistance during one-time uniaxial stretching}

Both types of the horseshoe-shaped interconnections discussed in the previous section were uniaxially stretched along the direction of interconnection using an Instron 5543 (figure 9). Together with an ohmmeter, this device allows for automated data collection (electrical resistance versus strain and time) of four interconnections simultaneously.

Figure 10 represents the course of the electrical resistance of each interconnection type during uniaxial stretching up to two times the original length (strain of $100 \%$ ) at a strain rate of $1 \% \mathrm{~s}^{-1}$. The initial electrical resistance of both tracks was $177 \Omega$. During stretching, the electrical resistance which is represented in $(b)$ shows minor variations. It has to be noted that the measurement precision of the setup is $\pm 7 \Omega$. The variations in $(b)$ thus fall within the measurement precision of the setup. It is quite clear though that the interconnection type which is represented by (a) suffers from a slight increase in electrical resistance. Optical microscopy after and even during stretching does not indicate why this increase occurs. Hence, we cannot yet elucidate this behaviour. It can be concluded that both types of interconnections stay highly conductive at strains up to $100 \%$. As the strain is released, the initial electrical resistance is recovered.

\subsection{Cyclic mechanical loading}

The lifetime of the horseshoe-shaped interconnections was evaluated by subjecting a limited number of fabricated test structures (two of each type) to various uniaxial strains for an extended period of time. The applied strains were chosen arbitrarily and thus solely serve as a means to demonstrate the cyclic mechanical performance of the interconnections. The strain rate was kept constant at $10 \% \mathrm{~s}^{-1}$ during the tests. The average number of cycles before electrical failure is graphically represented in figure 11 . 

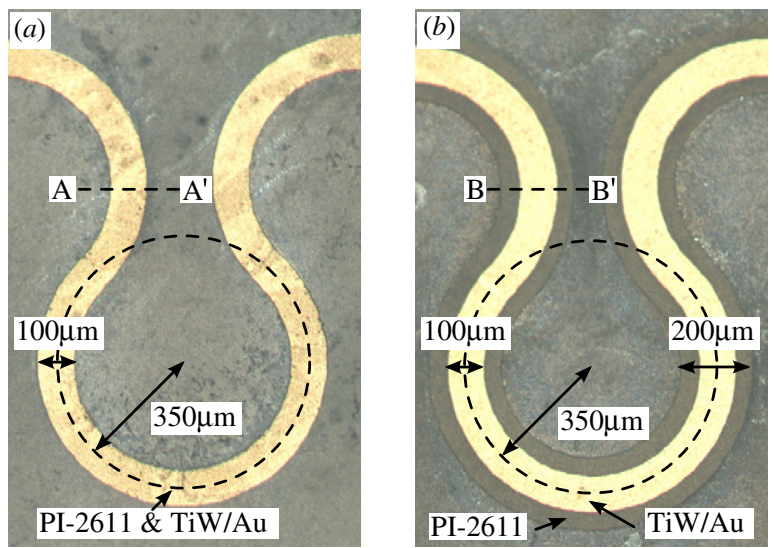

(c)
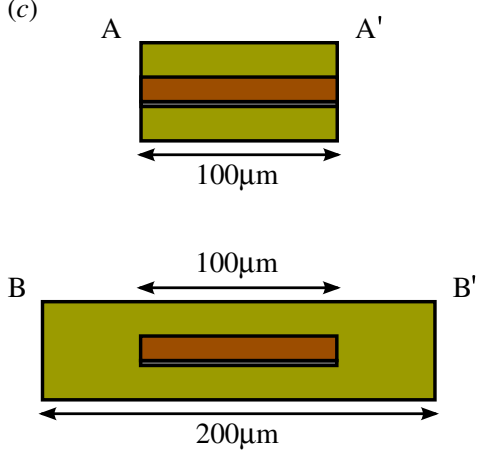

PI-2611 $\square \mathrm{Au} \square \mathrm{TiW}$

Figure 8. Zoomed view of horseshoe-shaped gold conductors (100 $\mu \mathrm{m}$ width) embedded in PDMS and supported by polyimide (100 $\mu \mathrm{m}$ width $(a)$ and $200 \mu \mathrm{m}$ width $(b))$. The indicated cross sections are visualized in $(c)$.

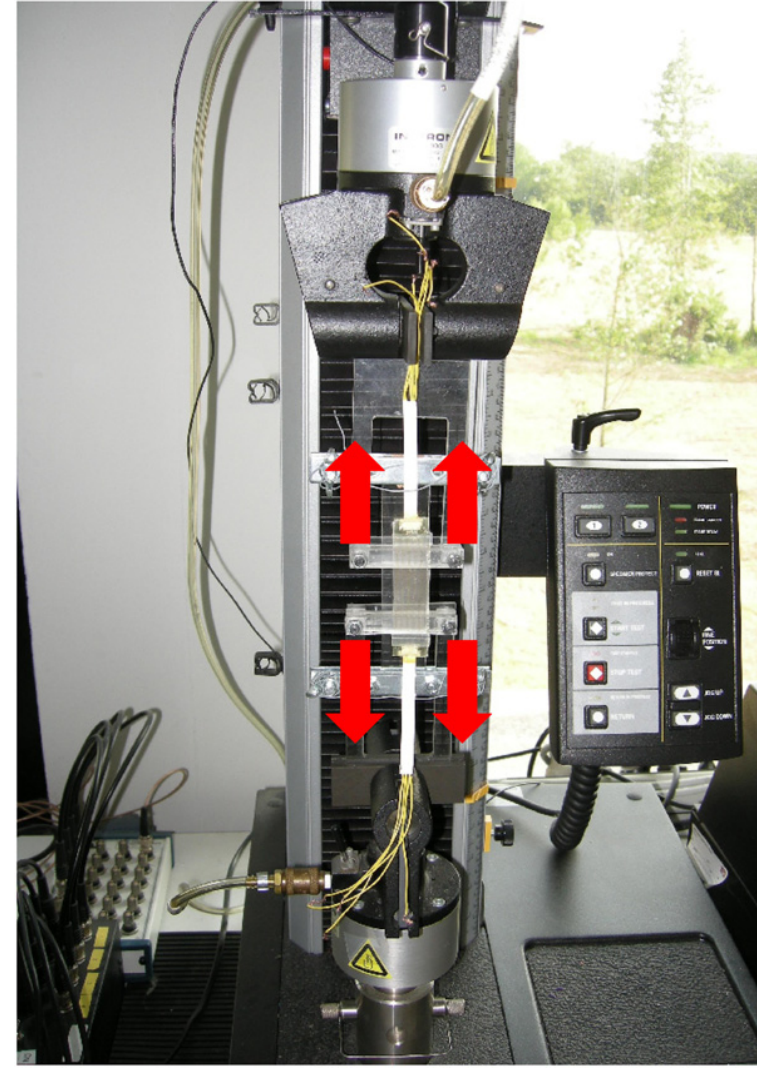

Figure 9. An Instron 5543 is used to uni-axially stretch the test structures.

From figure 11, it is clear that the width of the polyimide support has a great impact on the lifetime of the stretchable interconnections. This is clearly seen at the higher strains of $40 \%$ and $60 \%$ where the average number of cycles before electrical failure for conductors with the $200 \mu \mathrm{m}$ support width $(X)$ surpasses that of the conductors with $100 \mu \mathrm{m}$ support width $(Y)$, respectively by a factor $(X / Y) 170$ and 255 . It has to be noted that at these high strains, the PDMS in which the interconnections are embedded ruptured. The lifetime of the tracks with $200 \mu \mathrm{m}$ support width mentioned for these strains
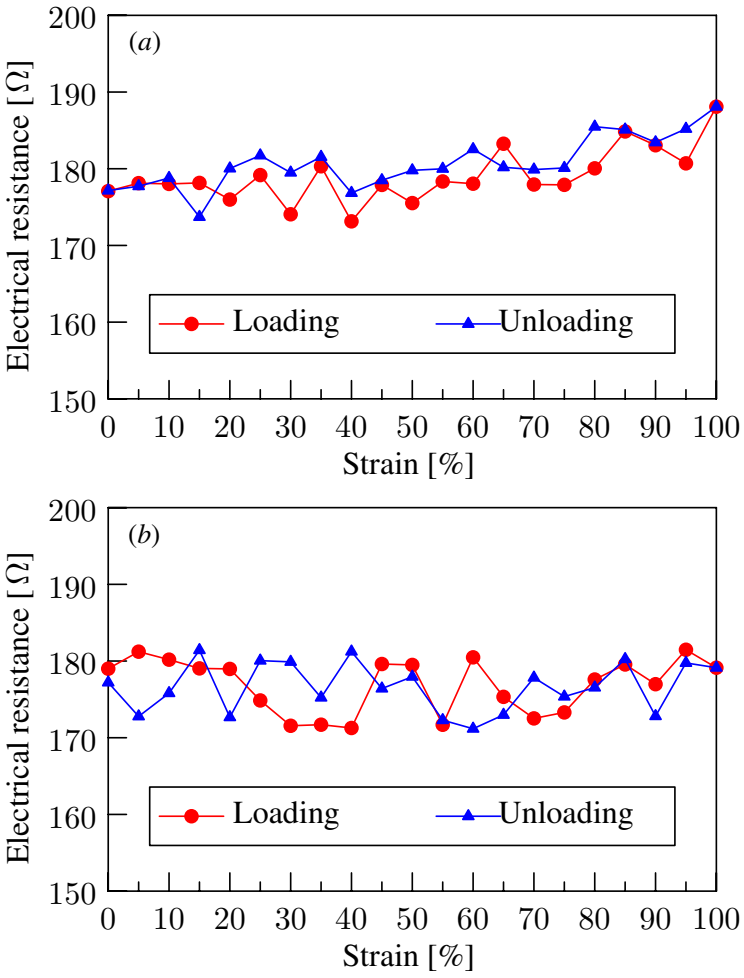

Figure 10. Electrical resistance of horseshoe-shaped interconnections with (a) $100 \mu \mathrm{m}$ and (b) $200 \mu \mathrm{m}$ polyimide support width, when uniaxially strained up to $100 \%$ at a strain rate of $1 \% \mathrm{~s}^{-1}$.

thus actually indicate electrical failure of the tracks due to rupture of the PDMS. This is attributed to the manual cutting of the test structures which introduced imperfections at the edges of the PDMS encapsulant.

At the lower strains, the interconnections have an increased lifetime. At $20 \%$ strain, a similar trend as for the higher strains is noticed; at $10 \%$ strain, both interconnection types have a lifetime exceeding 500000 cycles, with a hardly measurable change in resistance. The tests at these lower strains were eventually stopped at 500000 cycles without clear indication that electrical failure would occur. 


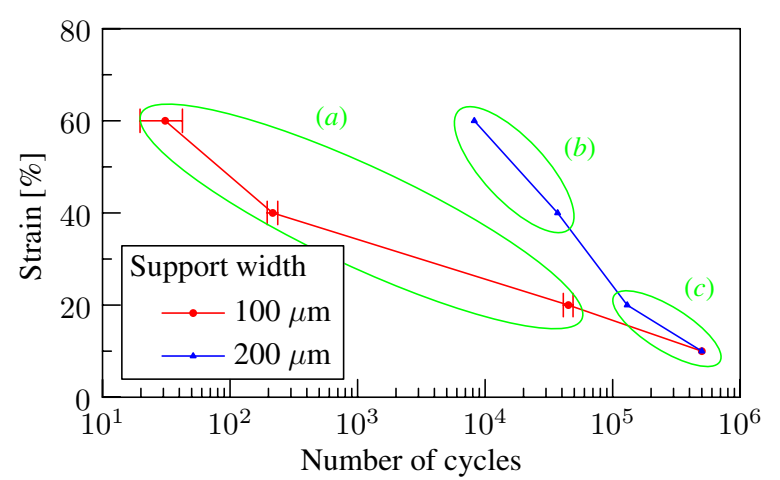

Figure 11. Average number of cycles before electrical failure of horseshoe-shaped conductors with varying polyimide support width when subjected to cyclic uniaxial strain. Three distinct regions are indicated: $(a)$ interconnections stopped conducting, $(b)$ rupture of the PDMS and $(c)$ interconnections still conducting when the test was stopped. The error bars represent the standard deviation $s$ of the sample consisting of two measurements.

\section{Conclusion}

In this paper, an architecture and new fabrication technology for stretchable electronics have been presented. The key aspect is the stretchable electrical interconnections, realized by patterning a $200 \mathrm{~nm}$ thick sputter-deposited gold film into meandering horseshoe shapes, and covered by polyimide PI-2611 as a means to improve the mechanical performance. The PI-2611 is patterned by dry etching; optimization through Taguchi methodology is illustrated.

The processing of the stretchable circuit is done on a rigid carrier; it is embedded in PDMS in a final stage. A customized release technique compatible with high temperatures up to $350{ }^{\circ} \mathrm{C}$ is developed based on the evaporation of a $400 \mathrm{~nm}$ thick layer of potassium chloride. Horseshoe-shaped conductors with a track width down to $20 \mu \mathrm{m}$ were embedded in PDMS. Furthermore, the technique allows for the use of different types of PDMS (e.g. biomedical grade). Since gold conductors are used, the technology could thus be applied for biomedical applications.

The technology was demonstrated by fabricating two versions of test structures consisting of parallel horseshoeshaped interconnections. These test structures consist of $100 \mu \mathrm{m}$ wide meandering gold conductors alternately covered by polyimide with a width of $100 \mu \mathrm{m}$ and $200 \mu \mathrm{m}$. For one version, conventional electronic components were mounted on separate polyimide islands which are interconnected by stretchable wiring and embedded into PDMS; the other version solely consists of stretchable interconnections. The resulting embedded circuits are both highly conformable and stretchable.

Reversible stretching of the test structures at uniaxial strains up to $100 \%$ was illustrated. Cyclic mechanical loading of test structures highlights the impact of the polyimide support width on the reliability. Stretchable interconnections composed of polyimide support which is $100 \mu \mathrm{m}$ wider than the gold conductor are found to have a greatly increased lifetime compared to interconnections for which the polyimide support and gold conductor have identical width. The former remained highly conductive at the applied strains until the PDMS ruptures, in contrast to the latter which fail at an earlier stage. Both interconnection types were found to have a lifetime of minimum 500000 cycles at a strain of $10 \%$. No change in electrical resistance was noticed.

\section{Acknowledgments}

The authors would like to thank the Agency for Innovation by Science and Technology in Flanders (IWT) for the financial support through the SBO-BrainSTAR project. This research is also supported by an individual IMEC PhD grant.

\section{References}

[1] Yu Z, Graudejus O, Tsay C, Lacour S P, Wagner S and Morrison B 2009 Monitoring hippocampus electrical activity in vitro on an elastically deformable microelectrode array J. Neurotraum. 26 1135-45

[2] Graudejus O, Yu Z, Jones J, Morrison B and Wagner S 2009 Characterization of an elastically stretchable microelectrode array and its application to neural field potential recordings J. Electrochem. Soc. 156 P85-94

[3] Wei P, Taylor R, Ding Z, Higgs G, Norman J J, Pruitt B L and Ziaie B 2009 A stretchable cell culture platform with embedded electrode array Proc. 22nd IEEE Int. Conf. on Micro Electro Mechanical Systems (Sorrento, 25-29 January 2009) pp 407-10

[4] Urdaneta M G, Delille R and Smela E 2007 Stretchable electrodes with high conductivity and photo-patternability Adv. Mater. 19 2629-33

[5] Sekitani T, Noguchi Y, Hata K, Fukushima T, Aida T and Someya $\mathrm{T} 2008$ A rubberlike stretchable active matrix using elastic conductors Science 321 1468-72

[6] Kim D H and Rogers J A 2008 Stretchable electronics: materials strategies and devices Adv. Mater. 20 4887-92

[7] Kim D H, Xiao J, Song J, Huang Y and Rogers J A 2010 Stretchable, curvilinear electronics based on inorganic materials Adv. Mater. 22 2108-24

[8] Kim D H et al 2011 Materials for multifunctional balloon catheters with capabilities in cardiac electrophysiological mapping and ablation therapy Nat. Mater 10 316-23

[9] Wagner S, Lacour S P, Jones J, Hsu P I, Sturm J C, Li T and Suo Z 2004 Electronic skin: architecture and components Physica E 25 326-34

[10] Lacour S P, Chan D, Wagner S, Li T and Suo Z 2006 Mechanisms of reversible stretchability of thin metal films on elastomeric substrates Appl. Phys. Lett. 88204103

[11] Graz I M, Cotton D P J and Lacour S P 2009 Extended cyclic uniaxial loading of stretchable gold thin-films on elastomeric substrates Appl. Phys. Lett. 94071902

[12] Gray D S, Tien J and Chen C S 2004 High-conductivity elastomeric electronics Adv. Mater. 16 393-97

[13] Brosteaux D, Axisa F, Gonzalez M and Vanfleteren J 2007 Design and fabrication of elastic interconnections for stretchable electronic circuits IEEE Electron Device Lett. 28 552-54

[14] Bossuyt F, Guenther J, Löher T, Seckel M, Sterken T and de Vries J 2011 Cyclic endurance reliability of stretchable electronic substrates Microelectron. Reliab. 51 628-35

[15] Christiaens W, Bosman E and Vanfleteren J 2010 UTCP: a novel polyimide based ultra-thin chip packaging technology IEEE Trans. Compon. Pack. Technol. 33 754-60

[16] Gonzalez M, Axisa F, Bulcke M V, Brosteaux D, Vandevelde B and Vanfleteren J 2008 Design of metal interconnects for stretchable electronic circuits Microelectron. Reliab. 48 825-32 
[17] Sterken T, Bossuyt F, Verplancke R, Vervust T, Axisa F and Vanfleteren J 2010 Lifetime of stretchable meander shaped copper conductors in PDMS subjected to cyclic elongation MRS Spring Meeting (San Francisco, CA, 5-9 April 2010)

[18] Hsu Y Y, Gonzalez M, Bossuyt F, Vanfleteren J and De Wolf I 2011 Polyimide-enhanced stretchable interconnects: design, fabrication and characterization IEEE Trans. Electron Devices $\mathbf{5 8} 2680-8$

[19] HD Microsystems Product Bulletin of PI-2600 series, available from http://hdmicrosystems.com/
[20] Metz S, Bertsch A and Renaud P 2005 Partial release and detachment of microfabricated metal and polymer structures by anodic metal dissolution $J$. Microelectromech Syst. 14 383-91

[21] Montgomery D C 2005 Design and Analysis of Experiments 6th edn (Arizona: Wiley) p 203

[22] Hsu Y Y, Gonzalez M, Bossuyt F, Axisa F, Vanfleteren J and De Wolf I 2010 The effect of pitch on deformation behavior and the stretching-induced failure of a polymer-encapsulated stretchable circuit $J$. Micromech. Microeng. 20075036 